\title{
Korobov polynomials of the third kind and of the fourth kind
}

\author{
Taekyun Kim ${ }^{1,2}$ and Dae San $\mathrm{Kim}^{3^{*}}$
}

*Correspondence:
dskim@sogang.ac.kr
${ }^{3}$ Department
of Mathematics, Sogang
University, Seoul 121-742,
Republic of Korea
Full list of author information
is available at the end of the
article

${ }^{*}$ Correspondence:

dskim@sogang.ac.k

of Mathematics, Sogang

University, Seoul 121-742,

Full list of author information article

\begin{abstract}
The first degenerate version of the Bernoulli polynomials of the second kind appeared in the paper by Korobov (Math Notes 2:77-19, 1996; Proceedings of the IVinternational conference modern problems of number theory and its applications, pp 40-49, 2001). In this paper, we study two degenerate versions of the Bernoulli polynomials of the second kind which will be called Korobov polynomials of third kind and of the fourth kind. Some properties, identities, recurrence relations and connections with other polynomials are investigated by using umbral calculus.
\end{abstract}

Keywords: Korobov polynomials of the third kind and of the fourth kind, umbral calculus

Mathematics subject classification: 05A19, 05A40, $11 \mathrm{~B} 83$

\section{Background}

The Bernoulli polynomials of the second kind $b_{n}(x)$ are given by the generating function

$$
\frac{t}{\log (1+t)}(1+t)^{x}=\sum_{n=0}^{\infty} b_{n}(x) \frac{t^{n}}{n !}, \quad \text { (see Kim et al. 2014, 2015; Roman 1984). }
$$

When $x=0, b_{n}=b_{n}(0)$ are called Bernoulli numbers of the second kind. The degenerate version of the Bernoulli polynomials of the second kind are called Korobov polynomials of the first kind. We note here that the Carlitz degerate Bernoulli polynomials were rediscovered by Ustinov under the name of Korobov polynomial of the second kind (see Pylypiv and Maliarchuk 2014; Ustinov 2003).

The Daehee polynomials $D_{n}(x)$ are defined by the generating function

$$
\frac{\log (1+t)}{t}(1+t)^{x}=\sum_{n=0}^{\infty} D_{n}(x) \frac{t^{n}}{n !}, \quad(\text { see Kim et al. 2014, 2015) }
$$

For $x=0, D_{n}=D_{n}(0)$ are called Daehee numbers.

The Korobov polynomials $K_{n}(\lambda, x)$ of the first kind are given by the generating function

$$
\frac{\lambda t}{(1+t)^{\lambda}-1}(1+t)^{x}=\sum_{n=0}^{\infty} K_{n}(x \mid \lambda) \frac{t^{n}}{n !}, \quad \text { (see Korobov 1996; Korobov 2001). }
$$

\section{包 Springer}

(c) 2015 Kim and Kim. This article is distributed under the terms of the Creative Commons Attribution 4.0 International License (http://creativecommons.org/licenses/by/4.0/), which permits unrestricted use, distribution, and reproduction in any medium, provided you give appropriate credit to the original author(s) and the source, provide a link to the Creative Commons license, and indicate if changes were made. 
When $x=0, K_{n}(\lambda)=K_{n}(0 \mid \lambda)$ are called Korobov numbers of the first kind.

In the following, we will review very briefly some necessary things on umbral calculus. Our basic reference is Roman (1984). Also, one is asked to look at more recent papers on umbral calculus (Nisar et al. 2015; Srivastava et al. 2014).

Let $\mathbb{C}$ be the complex number field and let $\mathcal{F}$ be the set of all formal power series in the variable $t$ over $\mathbb{C}$ with

$$
\mathcal{F}=\left\{f(t)=\sum_{k=0}^{\infty} \frac{a_{k}}{k !} t^{k} \mid a_{k} \in \mathbb{C}\right\}
$$

Let $\mathbb{P}=\mathbb{C}[x]$ and let $\mathbb{P}^{*}$ be the vector space of all linear functionals on $\mathbb{P}$. For $L \in \mathbb{P}^{*}$, the action of the linear functional $L$ on a polynomial $p(x)$ is denoted by $\langle L \mid p(x)\rangle$ with

$$
\langle L+M \mid p(x)\rangle=\langle L \mid p(x)\rangle+\langle M \mid p(x)\rangle, \quad\langle c L \mid p(x)\rangle=c\langle L \mid p(x)\rangle,
$$

where $c$ is a complex constant (see Kim 2014; Roman 1984).

For $f(t)=\sum_{k=0}^{\infty} a_{k} \frac{t^{k}}{k !} \in \mathcal{F}$, we define a linear functional on $\mathbb{P}$ by setting

$$
\left\langle f(t) \mid x^{n}\right\rangle=a_{n}, \quad \text { for all } n \geq 0, \quad \text { (see Kim et al. 2014; Roman 1984). }
$$

Thus, by (1.5), we easily get

$$
\left\langle t^{k} \mid x^{n}\right\rangle=n ! \delta_{n, k}, \quad(n, k \geq 0)
$$

where $\delta_{n, k}$ is the Kronecker's symbol.

Let $f_{L}(t)=\sum_{k=0}^{\infty}\left\langle L \mid x^{k}\right\rangle \frac{t^{n}}{k !}$. Then, by (1.6), we get $\left\langle f_{L}(t) \mid x^{n}\right\rangle=\left\langle L \mid x^{n}\right\rangle$. Additionallly, the mapping $L \mapsto f_{L}(t)$ is a vector space isomorphism from $\mathbb{P}^{*}$ onto $\mathcal{F}$. Henceforth, $\mathcal{F}$ denotes both the algebra of formal power series in $t$ and the vector space of all linear functionals on $\mathbb{P}$, and so an element $f(t)$ of $\mathcal{F}$ can be regarded as both a formal power series and a linear functional. We refer to $\mathcal{F}$ as the umbral algebra. The umbral calculus is the study of umbral algebra (see Kim 2014; Roman 1984). From (1.6), we can easily derive $\left\langle e^{y t} \mid x^{n}\right\rangle=y^{n}$. So $\left\langle e^{y t} \mid p(x)\right\rangle=p(y)$. The order $o(f(t))$ of a power series $f(t)(\neq 0)$ is the smallest nonnegative integer $k$ for which the coefficient at $t^{k}$ does not vanish. For $f(t) \in \mathcal{F}$ and $p(x) \in \mathbb{P}$, we have

$$
f(t)=\sum_{k=0}^{\infty}\left\langle f(t) \mid x^{k}\right\rangle \frac{t^{k}}{k !}, \quad p(x)=\sum_{k=0}^{\infty}\left\langle t^{k} \mid p(x)\right\rangle \frac{x^{k}}{k !} .
$$

Thus, by (1.7), we get

$$
p^{(k)}(0)=\left.\left(\frac{d}{d x}\right)^{k} p(x)\right|_{x=0}=\left\langle t^{k} \mid p(x)\right\rangle=\left\langle 1 \mid p^{(k)}(x)\right\rangle .
$$

From (1.8), we note that

$$
t^{k} p(x)=p^{(k)}(x)=\frac{d^{k}}{d x^{k}} p(x), \quad e^{y t} p(x)=p(x+y), \quad(\text { see Roman 1984). }
$$

Let $f(t), g(t) \in \mathcal{F}$ such that $o(f(t))=1$ and $o(g(t))=0$. Then there exists a unique sequence $s_{n}(x)\left(\operatorname{deg} s_{n}(x)=n\right)$ of polynomials such that $\left\langle g(t) f(t)^{k} \mid s_{n}(x)\right\rangle=n ! \delta_{n, k}$, for 
$n, k \geq 0$. The sequence $s_{n}(x)$ is called the Sheffer sequence for the pair $(g(t), f(t))$, which is denoted by $s_{n}(x) \sim(g(t), f(t))$. For $s_{n}(x) \sim(g(t), f(t))$, we have

$$
f(t) s_{n}(x)=n s_{n-1}(x), \quad(n \in \mathbb{N} \cup\{0\}),
$$

and

$$
\frac{1}{g(\bar{f}(t))} e^{x \bar{f}(t)}=\sum_{k=0}^{\infty} \frac{s_{k}(x)}{k !} t^{k}, \quad \text { for all } x \in \mathbb{C} .
$$

Here $\bar{f}(t)$ is the compositional inverse of $f(t)$ (see Kim and Mansour 2014; Roman 1984).

The conjugation representation for $s_{n}(x) \sim(g(t), f(t))$ is given by

$$
s_{n}(x)=\sum_{k=0}^{n} \frac{1}{k !}\left\langle g(\bar{f}(t))^{-1} \bar{f}(t)^{k} \mid x^{n}\right\rangle x^{k}, \quad(n \geq 0), \quad(\text { see Carlitz 1979; Roman 1984). }
$$

Let us consider the following two Sheffer sequences:

$$
s_{n}(x) \sim(g(t), f(t)), \quad r_{n}(x) \sim(h(t), l(t)) .
$$

Then, we have

$$
s_{n}(x)=\sum_{m=0}^{n} C_{n, m} r_{m}(x), \quad(n \geq 0)
$$

where

$$
C_{n, m}=\frac{1}{m !}\left\langle\frac{h(\bar{f}(t))}{g(\bar{f}(t))}(l(\bar{f}(t)))^{m} \mid x^{n}\right\rangle, \quad \text { (see Kim et al. 2014; Roman 1984). }
$$

The first degenerate version of the Bernoulli polynomials of the second kind appeared in the paper by Korobov $(2001 ; 1996)$. In this paper, we study two degenerate versions of the Bernoulli polynomials of the second kind which will be called Korobov polynomials of the third kind and of the fourth kind. Some properties, identities and recurrence relations for them are investigated by using umbral calculus. In addition, some connections with other polynomials are studied for which one refers to the related papers (Dattoli et al. 2006, 2004).

\section{Korobov polynomials of the third kind and of the fourth kind}

Now, we introduce Korobov polynomials of the third kind $K_{n, 3}(x \mid \lambda)$ and of the fourth kind $K_{n, 4}(x \mid \lambda)$, respectively, given by the generating functions

$$
\frac{\log (1+\lambda t)}{\lambda \log (1+t)}(1+t)^{x}=\sum_{n=0}^{\infty} K_{n, 3}(x \mid \lambda) \frac{t^{n}}{n !}
$$

and 


$$
\frac{\log (1+\lambda t)}{(1+t)^{\lambda}-1}(1+t)^{x}=\sum_{n=0}^{\infty} K_{n, 4}(x \mid \lambda) \frac{t^{n}}{n !}
$$

When $x=0, K_{n, 3}(\lambda)=K_{n, 3}(0, \lambda)$ and $K_{n, 4}(\lambda)=K_{n, 4}(0 \mid \lambda)$ are called Korobov numbers of the third kind and of the fourth kind, respectively.

$$
\text { As all } \frac{\lambda t}{(1+t)^{\lambda}-1}=\frac{t}{\frac{(1+t)^{\lambda}-1}{\lambda}}, \frac{\log (1+\lambda t)}{\lambda \log (1+t)}=\frac{\log (1+\lambda t)^{\frac{1}{\lambda}}}{\log (1+t)}, \frac{\log (1+\lambda t)}{(1+t)^{\lambda}-1}=\frac{\log (1+\lambda t)^{\frac{1}{\lambda}}}{\frac{(1+\lambda t)^{\lambda}-1}{\lambda}} \text { tend to } \frac{t}{\log (1+t)}
$$
as $\lambda \rightarrow 0, \lim _{\lambda \rightarrow 0} K_{n}(x \mid \lambda)=\lim _{\lambda \rightarrow 0} K_{n, 3}(x \mid \lambda)=\lim _{\lambda \rightarrow 0} K_{n, 4}(x \mid \lambda)=b_{n}(x), \quad(n \geq 0)$. We observe first that $K_{n, 3}(x \mid \lambda)$ and $K_{n, 4}(x \mid \lambda)$ are Sheffer sequences for the respective pairs $\left(\frac{\lambda t}{\log \left(1+\lambda\left(e^{t}-1\right)\right)}, e^{t}-1\right)$ and $\left(\frac{e^{\lambda t}-1}{\log \left(1+\lambda\left(e^{t}-1\right)\right)}, e^{t}-1\right)$. That is,

$$
K_{n, 3}(x \mid \lambda) \sim\left(\frac{\lambda t}{\log \left(1+\lambda\left(e^{t}-1\right)\right)}, e^{t}-1\right),
$$

and

$$
K_{n, 4}(x \mid \lambda) \sim\left(\frac{e^{\lambda t}-1}{\log \left(1+\lambda\left(e^{t}-1\right)\right)}, e^{t}-1\right) .
$$

From (1.12) and (2.2), we have

$$
K_{n, 3}(x \mid \lambda)=\sum_{k=0}^{n} \frac{1}{k !}\left\langle\frac{\log (1+\lambda t)}{\lambda \log (1+t)}(\log (1+t))^{k} \mid x^{n}\right\rangle x^{k} .
$$

We observe that

$$
\begin{aligned}
\langle & \frac{\log (1+\lambda t)}{\lambda \log (1+t)}(\log (1+x))^{k}\left|x^{n}\right\rangle \\
= & \left\langle\frac{\log (1+\lambda t)}{\lambda \log (1+t)} \mid(\log (1+x))^{k} x^{n}\right\rangle \\
= & \left\langle\frac{\log (1+\lambda t)}{\lambda \log (1+t)} \mid k ! \sum_{l=k}^{\infty} S_{1}(l, k) \frac{t^{l}}{l !} x^{n}\right\rangle \\
= & k ! \sum_{l=k}^{n}\left(\begin{array}{c}
n \\
l
\end{array}\right) S_{1}(l, k)\left\langle\frac{\log (1+\lambda t)}{\lambda t} \mid \frac{t}{\log (1+t)} x^{n-l}\right\rangle \\
= & k ! \sum_{l=k}^{n}\left(\begin{array}{c}
n \\
l
\end{array}\right) S_{1}(l, k)\left\langle\frac{\log (1+\lambda t)\left|\sum_{m=0}^{\infty} b_{m} \frac{t^{m}}{m !} x^{n-l}\right\rangle}{\lambda t}\right\rangle \\
= & k ! \sum_{l=k}^{n}\left(\begin{array}{c}
n \\
l
\end{array}\right) S_{1}(l, k) \sum_{m=0}^{n-l}\left(\begin{array}{c}
n-l \\
m
\end{array}\right) b_{m}\left\langle\frac{\log (1+\lambda t)}{\lambda t} \mid x^{n-l-m}\right\rangle \\
= & k ! \sum_{l=k}^{n}\left(\begin{array}{c}
n \\
l
\end{array}\right) S_{1}(l, k) \sum_{m=0}^{n-l}\left(\begin{array}{c}
n-l \\
m
\end{array}\right) b_{m}\left\langle\sum_{j=0}^{\infty} D_{j} \lambda^{j} \frac{t^{j}}{j !} \mid x^{n-l-m}\right\rangle \\
= & k ! \sum_{l=k}^{n}\left(\begin{array}{c}
n \\
l
\end{array}\right) S_{1}(l, k) \sum_{m=0}^{n-l}\left(\begin{array}{c}
n-l \\
m
\end{array}\right) b_{m} D_{n-l-m} \lambda^{n-l-m} \\
= & k ! \sum_{l=k}^{n} \sum_{m=0}^{n-l}\left(\begin{array}{c}
n \\
l
\end{array}\right)\left(\begin{array}{c}
n-l \\
m
\end{array}\right) S_{1}(l, k) b_{m} D_{n-l-m} \lambda^{n-l-m},
\end{aligned}
$$


where $S_{1}(n, m)$ is the Stirling number of the first kind defined by

$$
x(x-1) \ldots(x-n+1)=(x)_{n}=\sum_{l=0}^{n} S_{1}(n, l) x^{l}, \quad(n \geq 0) .
$$

Therefore, by (2.4) and (2.5), we have

Theorem 1 For $n \geq 0$, we have

$$
K_{n, 3}(x \mid \lambda)=\sum_{k=0}^{n}\left(\sum_{l=k}^{n} \sum_{m=0}^{n-l}\left(\begin{array}{c}
n \\
l
\end{array}\right)\left(\begin{array}{c}
n-l \\
m
\end{array}\right) S_{1}(l, k) b_{n-l-m} D_{m} \lambda^{m}\right) x^{k} .
$$

From (1.12) and (2.3), we have

$$
K_{n, 4}(x \mid \lambda)=\sum_{k=0}^{n} \frac{1}{k !}\left\langle\frac{\log (1+\lambda t)}{(1+t)^{\lambda}-1}(\log (1+t))^{k} \mid x^{n}\right\rangle x^{k} .
$$

We observe that

$$
\begin{aligned}
& \left\langle\frac{\log (1+\lambda t)}{(1+t)^{\lambda}-1}(\log (1+t))^{k} \mid x^{n}\right\rangle \\
& =k ! \sum_{l=k}^{n}\left(\begin{array}{c}
n \\
l
\end{array}\right) S_{1}(l, k)\left\langle\frac{\log (1+\lambda t)}{\lambda t} \frac{\lambda t}{(1+t)^{\lambda}-1} \mid x^{n-l}\right\rangle \\
& =k ! \sum_{l=k}^{n}\left(\begin{array}{c}
n \\
l
\end{array}\right) S_{1}(l, k)\left\langle\frac{\log (1+\lambda t)}{\lambda t} \mid \sum_{m=0}^{\infty} K_{m}(\lambda) \frac{t^{m}}{m !} x^{n-l}\right\rangle \\
& =k ! \sum_{l=k}^{n}\left(\begin{array}{c}
n \\
l
\end{array}\right) S_{1}(l, k) \sum_{m=0}^{n-l}\left(\begin{array}{c}
n-l \\
m
\end{array}\right) K_{m}(\lambda)\left\langle\frac{\log (1+\lambda t)}{\lambda t} \mid x^{n-l-m}\right\rangle \\
& =k ! \sum_{l=k}^{n}\left(\begin{array}{c}
n \\
l
\end{array}\right) S_{1}(l, k) \sum_{m=0}^{n-l}\left(\begin{array}{c}
n-l \\
m
\end{array}\right) K_{m}(\lambda) D_{n-l-m} \lambda^{n-l-m} \\
& =k ! \sum_{l=k}^{n} \sum_{m=0}^{n-l}\left(\begin{array}{c}
n \\
l
\end{array}\right)\left(\begin{array}{c}
n-l \\
m
\end{array}\right) S_{1}(l, k) K_{n-l-m}(\lambda) D_{m} \lambda^{m} .
\end{aligned}
$$

Therefore, by (2.6) and (2.7), we obtain the following theorem.

Theorem 2 For $n \geq 0$, we have

$$
K_{n, 4}(x \mid \lambda)=\sum_{k=0}^{n}\left(\sum_{l=k}^{n} \sum_{m=0}^{n-l}\left(\begin{array}{c}
n \\
l
\end{array}\right)\left(\begin{array}{c}
n-l \\
m
\end{array}\right) S_{1}(l, k) K_{n-l-m}(\lambda) D_{m} \lambda^{m}\right) x^{k} .
$$

By (1.6) and (2.1), we easily get 


$$
\begin{aligned}
K_{n, 3}(y \mid \lambda) & =\left\langle\frac{\log (1+\lambda t)}{\lambda \log (1+t)}(1+t)^{y} \mid x^{n}\right\rangle \\
& =\left\langle\frac{\log (1+\lambda t)}{\lambda \log (1+t)} \mid(1+t)^{y} x^{n}\right\rangle \\
& =\left\langle\frac{\log (1+\lambda t)}{\lambda \log (1+t)} \mid \sum_{l=0}^{\infty}(y)_{l} \frac{t^{l}}{l !} x^{n}\right\rangle \\
& =\sum_{l=0}^{n}\left(\begin{array}{c}
n \\
l
\end{array}\right)(y)_{l}\left\langle\frac{\log (1+\lambda t)}{\lambda \log (1+t)} \mid x^{n-l}\right\rangle \\
& =\sum_{l=0}^{n}\left(\begin{array}{c}
n \\
l
\end{array}\right)(y)_{l} \sum_{m=0}^{n-l}\left(\begin{array}{c}
n-l \\
m
\end{array}\right) b_{m} D_{n-l-m} \lambda^{n-l-m} \\
& =\sum_{l=0}^{n}\left(\begin{array}{c}
n \\
l
\end{array}\right)(y)_{l} \sum_{m=0}^{n-l}\left(\begin{array}{c}
n-l \\
m
\end{array}\right) b_{n-l-m} D_{m} \lambda^{m} \\
& =\sum_{l=0}^{n}\left(\sum_{m=0}^{n-l}\left(\begin{array}{c}
n \\
l
\end{array}\right)\left(\begin{array}{c}
n-l \\
m
\end{array}\right) b_{n-l-m} D_{m} \lambda^{m}\right)(y)_{l} .
\end{aligned}
$$

Thus, by (2.8), we get

$$
K_{n, 3}(x \mid \lambda)=\sum_{l=0}^{n}\left(\sum_{m=0}^{n-l}\left(\begin{array}{c}
n \\
l
\end{array}\right)\left(\begin{array}{c}
n-l \\
m
\end{array}\right) b_{n-l-m} D_{m} \lambda^{m}\right)(x)_{l}, \quad(n \geq 0) .
$$

From (1.6) and (2.2), we note that

$$
\begin{aligned}
& K_{n, 4}(y \mid \lambda)=\left\langle\frac{\log (1+\lambda t)}{(1+t)^{\lambda}-1}(1+t)^{y} \mid x^{n}\right\rangle \\
& =\left\langle\frac{\log (1+\lambda t)}{(1+t)^{\lambda}-1} \mid(1+t)^{y} x^{n}\right\rangle \\
& =\sum_{l=0}^{n}\left(\begin{array}{c}
n \\
l
\end{array}\right)(y)_{l}\left\langle\frac{\log (1+\lambda t)}{(1+t)^{\lambda}-1} \mid x^{n-l}\right\rangle \\
& =\sum_{l=0}^{n}\left(\begin{array}{l}
n \\
l
\end{array}\right)(y)_{l} \sum_{m=0}^{n-l}\left(\begin{array}{c}
n-l \\
m
\end{array}\right) K_{m}(\lambda) D_{n-l-m} \lambda^{n-l-m} \\
& =\sum_{l=0}^{n}\left(\begin{array}{c}
n \\
l
\end{array}\right)(y)_{l} \sum_{m=0}^{n-l}\left(\begin{array}{c}
n-l \\
m
\end{array}\right) K_{n-l-m}(\lambda) D_{m} \lambda^{m} \\
& =\sum_{l=0}^{n}\left(\sum_{m=0}^{n-l}\left(\begin{array}{c}
n \\
l
\end{array}\right)\left(\begin{array}{c}
n-l \\
m
\end{array}\right) K_{n-l-m}(\lambda) D_{m} \lambda^{m}\right)(y)_{l} \text {. }
\end{aligned}
$$

Thus, by (2.10), we get

$$
K_{n, 4}(x \mid \lambda)=\sum_{l=0}^{n}\left(\sum_{m=0}^{n-l}\left(\begin{array}{c}
n \\
l
\end{array}\right)\left(\begin{array}{c}
n-l \\
m
\end{array}\right) K_{n-l-m}(\lambda) D_{m} \lambda^{m}\right)(x)_{l} .
$$

From (2.2), we note that 


$$
\begin{aligned}
K_{n, 3}(x \mid \lambda) \sim\left(\frac{\lambda t}{\log \left(1+\lambda\left(e^{t}-1\right)\right)}, e^{t}-1\right) \\
\Longleftrightarrow \frac{\lambda t}{\log \left(1+\lambda\left(e^{t}-1\right)\right)} K_{n, 3}(x \mid \lambda)=(x)_{n} \sim\left(1, e^{t}-1\right) .
\end{aligned}
$$

By (2.12), we get

$$
\begin{aligned}
K_{n, 3}(x \mid \lambda) & =\frac{\log \left(1+\lambda\left(e^{t}-1\right)\right)}{\lambda t}(x)_{n} \\
& =\sum_{k=0}^{n} S_{1}(n, k) \frac{\log \left(1+\lambda\left(e^{t}-1\right)\right)}{\lambda t} x^{k} \\
& =\sum_{k=0}^{n} S_{1}(n, k) \frac{e^{t}-1}{t} \frac{\log \left(1+\lambda\left(e^{t}-1\right)\right)}{\lambda\left(e^{t}-1\right)} x^{k} \\
& =\sum_{k=0}^{n} S_{1}(n, k) \frac{e^{t}-1}{t} \sum_{l=0}^{k} D_{l} \lambda^{l} \frac{\left(e^{t}-1\right)^{l}}{l !} x^{k} \\
& =\sum_{k=0}^{n} S_{1}(n, k) \frac{e^{t}-1}{t} \sum_{l=0}^{k} D_{l} \lambda^{l} \sum_{m=l}^{\infty} S_{2}(m, l) \frac{t^{m}}{m !} x^{k} \\
& =\sum_{k=0}^{n} S_{1}(n, k) \sum_{l=0}^{k} D_{l} \lambda^{l} \sum_{m=l}^{k}\left(\begin{array}{c}
k \\
m
\end{array}\right) S_{2}(m, l) \frac{e^{t}-1}{t} x^{k-m} .
\end{aligned}
$$

We observe that

$$
\begin{aligned}
\frac{e^{t}-1}{t} x^{k-m} & =\sum_{j=1}^{\infty} \frac{t^{j-1}}{j !} x^{k-m} \\
& =\sum_{j=0}^{\infty} \frac{1}{(j+1) !} t^{j} x^{k-m} \\
& =\sum_{j=0}^{k-m} \frac{1}{(j+1) !} t^{j} x^{k-m} \\
& =\sum_{j=0}^{k-m} \frac{1}{j+1}\left(\begin{array}{c}
k-m \\
j
\end{array}\right) x^{k-m-j} \\
& =\sum_{j=0}^{k-m} \frac{1}{k-m-j+1}\left(\begin{array}{c}
k-m \\
j
\end{array}\right) x^{j} .
\end{aligned}
$$

Thus, by (2.13) and (2.14), we have

$$
\begin{aligned}
& K_{n, 3}(x \mid \lambda) \\
& =\sum_{k=0}^{n} \sum_{l=0}^{k} \sum_{m=l}^{k} \sum_{j=0}^{k-m} \frac{1}{k-m-j+1}\left(\begin{array}{c}
k \\
m
\end{array}\right)\left(\begin{array}{c}
k-m \\
j
\end{array}\right) S_{1}(n, k) S_{2}(m, l) D_{l} \lambda^{l} x^{j} \\
& =\sum_{k=0}^{n} \sum_{l=0}^{k} \sum_{m=0}^{k-l} \sum_{j=0}^{m} \frac{1}{m-j+1}\left(\begin{array}{c}
k \\
m
\end{array}\right)\left(\begin{array}{c}
m \\
j
\end{array}\right) S_{1}(n, k) S_{2}(k-m, l) D_{l} \lambda^{l} x^{j} \\
& =\sum_{j=0}^{n}\left(\sum_{k=j}^{n} \sum_{l=0}^{k-j} \sum_{m=j}^{k-l} \frac{1}{k+1}\left(\begin{array}{c}
k+1 \\
m+1
\end{array}\right)\left(\begin{array}{c}
m+1 \\
j
\end{array}\right) S_{1}(n, k) S_{2}(k-m, l) D_{l} \lambda^{l}\right) x^{j},
\end{aligned}
$$


where $S_{2}(n, k)$ is the Stirling number of the second kind given by

$$
x^{n}=\sum_{l=0}^{n} S_{2}(n, l)(x)_{l}, \quad(n \geq 0) .
$$

Therefore, by (2.15), we obtain the following theorem expressing $K_{n, 3}(x \mid \lambda)$ in terms of the Stirling numbers of the first kind and of the second and Daehee numbers.

Theorem 3 For $n \geq 0$, we have

$$
\begin{aligned}
& K_{n, 3}(x \mid \lambda) \\
& =\sum_{j=0}^{n}\left(\sum_{k=j}^{n} \sum_{l=0}^{k-j} \sum_{m=j}^{k-l} \frac{1}{k+1}\left(\begin{array}{c}
k+1 \\
m+1
\end{array}\right)\left(\begin{array}{c}
m+1 \\
j
\end{array}\right) S_{1}(n, k) S_{2}(k-m, l) D_{l} \lambda^{l}\right) x^{j} .
\end{aligned}
$$

From (2.3), we have

$$
\frac{e^{\lambda t}-1}{\log \left(1+\lambda\left(e^{t}-1\right)\right)} K_{n, 4}(x \mid \lambda)=(x)_{n} \sim\left(1, e^{t}-1\right), \quad(n \geq 0) .
$$

Thus, by (2.16), we get

$$
\begin{aligned}
K_{n, 4}(x \mid \lambda) & =\frac{\log \left(1+\lambda\left(e^{t}-1\right)\right)}{e^{\lambda t}-1}(x)_{n} \\
& =\sum_{k=0}^{n} S_{1}(n, k) \frac{\log \left(1+\lambda\left(e^{t}-1\right)\right)}{e^{\lambda t}-1} x^{k} \\
& =\sum_{k=0}^{n} S_{1}(n, k) \frac{\lambda\left(e^{t}-1\right)}{e^{\lambda t}-1} \frac{\log \left(1+\lambda\left(e^{t}-1\right)\right)}{\lambda\left(e^{t}-1\right)} x^{k} \\
& =\sum_{k=0}^{n} S_{1}(n, k) \sum_{l=0}^{k} D_{l} \lambda^{l} \sum_{m=l}^{k}\left(\begin{array}{c}
k \\
m
\end{array}\right) S_{2}(m, l) \frac{e^{t}-1}{t} \frac{\lambda t}{e^{\lambda t}-1} x^{k-m},
\end{aligned}
$$

Now, we observe that

$$
\begin{aligned}
& \frac{e^{t}-1}{t} \frac{\lambda t}{e^{\lambda t}-1} x^{k-m}=\frac{e^{t}-1}{t} \sum_{j=0}^{k-m} B_{j} \lambda^{i} \frac{t^{j}}{j !} x^{k-m} \\
& =\sum_{j=0}^{k-m}\left(\begin{array}{c}
k-m \\
j
\end{array}\right) B_{j} \lambda^{j} \frac{e^{t}-1}{t} x^{k-m-j} \\
& =\sum_{j=0}^{k-m}\left(\begin{array}{c}
k-m \\
j
\end{array}\right) B_{j} \lambda^{j} \sum_{i=0}^{k-m-j} \frac{1}{i+1}\left(\begin{array}{c}
k-m-j \\
i
\end{array}\right) x^{k-m-j-i},
\end{aligned}
$$

where $B_{n}$ is the $n$-th Bernoulli number given by the generating function

$$
\frac{t}{e^{t}-1}=\sum_{n=0}^{\infty} B_{n} \frac{t^{n}}{n !}, \quad \text { (see Bayad and kim 2010; Kim et al. 2012, 2015). }
$$


Thus, by (2.17) and (2.18), we get

$$
\begin{aligned}
K_{n, 4}( & x \mid \lambda) \\
= & \sum_{k=0}^{n} S_{1}(n, k) \sum_{l=0}^{k} D_{l} \lambda^{l} \sum_{m=0}^{k-l}\left(\begin{array}{c}
k \\
m
\end{array}\right) S_{2}(k-m, l) \\
& \times \sum_{j=0}^{m}\left(\begin{array}{c}
m \\
j
\end{array}\right) B_{m-j} \lambda^{m-j} \sum_{i=0}^{j} \frac{1}{j-i+1}\left(\begin{array}{c}
j \\
i
\end{array}\right) x^{i} \\
= & \sum_{i=0}^{n}\left(\begin{array}{c}
n \\
k=i
\end{array} \sum_{l=0}^{k-i} \sum_{m=i}^{k-l} \sum_{j=i}^{m} \frac{1}{j-i+1}\left(\begin{array}{c}
k \\
m
\end{array}\right)\right. \\
& \left.\times\left(\begin{array}{c}
m \\
j
\end{array}\right)\left(\begin{array}{c}
j \\
i
\end{array}\right) \lambda^{l+m-j} S_{1}(n, k) S_{2}(k-m, l) D_{l} B_{m-j}\right) x^{i} \\
= & \sum_{i=0}^{n}\left(\begin{array}{c}
n \\
k-i
\end{array} \sum_{k=i}^{k-l} \sum_{l=0}^{m} \sum_{m=i}^{m} \frac{1}{k+1}\left(\begin{array}{c}
k+1 \\
m+1
\end{array}\right)\left(\begin{array}{c}
m+1 \\
j+1
\end{array}\right)\left(\begin{array}{c}
j+1 \\
i
\end{array}\right) \lambda^{l+m-j}\right. \\
& \left.\times S_{1}(n, k) S_{2}(k-m, l) D_{l} B_{m-j}\right) x^{i} .
\end{aligned}
$$

Therefore, by (2.19), we obtain the following theorem expressing $K_{n, 4}(x \mid \lambda)$ in terms of the Stirling numbers of the first kind and of the second kind, Daehee numbers and Bernoulli numbers.

Theorem 4 For $n \geq 0$, we have

$$
\begin{aligned}
& K_{n, 4}(x \mid \lambda) \\
& =\sum_{i=0}^{n}\left(\sum_{k=i}^{n} \sum_{l=0}^{k-i} \sum_{m=i}^{k-l} \sum_{j=i}^{m} \frac{1}{k+1}\left(\begin{array}{c}
k+1 \\
m+1
\end{array}\right)\left(\begin{array}{c}
m+1 \\
j+1
\end{array}\right)\left(\begin{array}{c}
j+1 \\
i
\end{array}\right)\right. \\
& \left.\quad \times \lambda^{l+m-j} S_{1}(n, k) S_{2}(k-m, l) D_{l} B_{m-j}\right) x^{i}
\end{aligned}
$$

From (2.8), we have

$$
\begin{aligned}
K_{n, 3}(y \mid \lambda) & =\sum_{l=0}^{n}\left(\begin{array}{c}
n \\
l
\end{array}\right)(y)_{l}\left\langle\frac{\log (1+\lambda t)}{\lambda \log (1+t)} \mid x^{n-l}\right\rangle \\
& =\sum_{l=0}^{n}\left(\begin{array}{l}
n \\
l
\end{array}\right)(y)_{l}\left\langle\sum_{i=0}^{\infty} K_{i, 3}(\lambda) \frac{t^{i}}{i !} \mid x^{n-l}\right\rangle \\
& =\sum_{l=0}^{n}\left(\begin{array}{l}
n \\
l
\end{array}\right)(y)_{l} K_{n-l, 3}(\lambda) .
\end{aligned}
$$

Thus, by (2.20), we get

$$
K_{n, 3}(x \mid \lambda)=\sum_{l=0}^{n}\left(\begin{array}{l}
n \\
l
\end{array}\right) K_{n-l, 3}(\lambda)(x)_{l} .
$$

From (2.10), we have 


$$
\begin{aligned}
K_{n, 4}(y \mid \lambda) & =\sum_{l=0}^{n}\left(\begin{array}{l}
n \\
l
\end{array}\right)(y)_{l}\left\langle\frac{\log (1+\lambda t)}{(1+t)^{\lambda}-1} \mid x^{n-l}\right\rangle \\
& =\sum_{l=0}^{n}\left(\begin{array}{l}
n \\
l
\end{array}\right)(y)_{l}\left\langle\sum_{i=0}^{\infty} K_{i, 4}(\lambda) \frac{t^{i}}{i !} \mid x^{n-l}\right\rangle \\
& =\sum_{l=0}^{n}\left(\begin{array}{l}
n \\
l
\end{array}\right)(y)_{l} K_{n-l, 4}(\lambda) .
\end{aligned}
$$

By (2.22), we get

$$
K_{n, 4}(x \mid \lambda)=\sum_{l=0}^{n}\left(\begin{array}{c}
n \\
l
\end{array}\right) K_{n-l, 4}(\lambda)(x)_{l}, \quad(n \geq 0) .
$$

From (2.19), we have

$$
\begin{aligned}
K_{n, 3}(y \mid \lambda) & =\left\langle\frac{\log (1+\lambda t)}{\lambda \log (1+t)}(1+t)^{y} \mid x^{n}\right\rangle \\
& =\left\langle\frac{\log (1+\lambda t)}{\lambda t} \mid \frac{t}{\log (1+t)}(1+t)^{y} x^{n}\right\rangle \\
& =\sum_{l=0}^{n}\left(\begin{array}{c}
n \\
l
\end{array}\right) b_{l}(y)\left\langle\frac{\log (1+\lambda t)}{\lambda t} \mid x^{n-l}\right\rangle \\
& =\sum_{l=0}^{n}\left(\begin{array}{c}
n \\
l
\end{array}\right) b_{l}(y)\left\langle\sum_{m=0}^{\infty} D_{m} \lambda^{m} \frac{t^{m}}{m !} \mid x^{n-l}\right\rangle \\
& =\sum_{l=0}^{n}\left(\begin{array}{c}
n \\
l
\end{array}\right) b_{l}(y) D_{n-l} \lambda^{n-l} .
\end{aligned}
$$

Thus, by (2.24), we get

$$
K_{n, 3}(x \mid \lambda)=\sum_{l=0}^{n}\left(\begin{array}{l}
n \\
l
\end{array}\right) D_{n-l} \lambda^{n-l} b_{l}(x), \quad(n \geq 0) .
$$

From (2.10), we can also derive the following equation:

$$
\begin{aligned}
K_{n, 4}(x \mid \lambda) & =\left\langle\frac{\log (1+\lambda t)}{(1+t)^{\lambda}-1}(1+t)^{y} \mid x^{n}\right\rangle \\
& =\left\langle\frac{\log (1+\lambda t)}{\lambda t} \mid \frac{\lambda t}{(1+t)^{\lambda}-1}(1+t)^{y} x^{n}\right\rangle \\
& =\left\langle\frac{\log (1+\lambda t)}{\lambda t} \mid \sum_{l=0}^{\infty} K_{l}(y \mid \lambda) \frac{t^{l}}{l !} x^{n}\right\rangle \\
& =\sum_{l=0}^{n}\left(\begin{array}{c}
n \\
l
\end{array}\right) K_{l}(y \mid \lambda)\left\langle\frac{\log (1+\lambda t)}{\lambda t} \mid x^{n-l}\right\rangle \\
& =\sum_{l=0}^{n}\left(\begin{array}{c}
n \\
l
\end{array}\right) K_{l}(y \mid \lambda)\left\langle\sum_{m=0}^{\infty} D_{m} \lambda^{m} \frac{t^{m}}{m !} \mid x^{n-l}\right\rangle \\
& =\sum_{l=0}^{n}\left(\begin{array}{c}
n \\
l
\end{array}\right) K_{l}(y \mid \lambda) D_{n-l} \lambda^{n-l} .
\end{aligned}
$$


Thus, by (2.26), we get

$$
K_{n, 4}(x \mid \lambda)=\sum_{l=0}^{n}\left(\begin{array}{c}
n \\
l
\end{array}\right) D_{n-l} \lambda^{n-l} K_{l}(x \mid \lambda) .
$$

Therefore, by (2.21), (2.23), (2.25) and (2.27), we obtain the following theorem expressing $K_{n, 3}(x \mid \lambda)$ and $K_{n, 4}(x \mid \lambda)$ both in terms of falling factorial polynomials. Also, we express $K_{n, 3}(x \mid \lambda)$ and $K_{n, 4}(x \mid \lambda)$ respectively by Bernoulli polynomials of the second kind and Korobov polynomials of the first kind.

Theorem 5 For $n \geq 0$, we have

$$
K_{n, 3}(x \mid \lambda)=\sum_{l=0}^{n}\left(\begin{array}{c}
n \\
l
\end{array}\right) K_{n-l, 3}(\lambda)(x)_{l}=\sum_{l=0}^{n}\left(\begin{array}{c}
n \\
l
\end{array}\right) D_{n-l} \lambda^{n-l} b_{l}(x),
$$

and

$$
K_{n, 4}(x \mid \lambda)=\sum_{l=0}^{n}\left(\begin{array}{c}
n \\
l
\end{array}\right) K_{n-l, 4}(\lambda)(x)_{l}=\sum_{l=0}^{n}\left(\begin{array}{c}
n \\
l
\end{array}\right) D_{n-l} \lambda^{n-l} K_{l}(x \mid \lambda) .
$$

It is easy to see that

$$
x^{n} \sim(1, t), \quad \frac{\lambda t}{\log \left(1+\lambda\left(e^{t}-1\right)\right)} K_{n, 3}(x \mid \lambda) \sim\left(1, e^{t}-1\right) .
$$

For $n \geq 1$, we have

$$
\begin{aligned}
\frac{\lambda t}{\log \left(1+\lambda\left(e^{t}-1\right)\right)} K_{n, 3}(x \mid \lambda) & =x\left(\frac{t}{e^{t}-1}\right)^{n} x^{-1} x^{n} \\
& =x B_{n-1}^{(n)}(x) \\
& =\sum_{k=0}^{n-1}\left(\begin{array}{c}
n-1 \\
k
\end{array}\right) B_{k}^{(n)} x^{n-k} \\
& =\sum_{k=1}^{n}\left(\begin{array}{c}
n-1 \\
k-1
\end{array}\right) B_{n-k}^{(n)} x^{k} .
\end{aligned}
$$

Thus, by (2.29), we get

$$
\begin{aligned}
K_{n, 3}(x \mid \lambda) \\
=\sum_{k=1}^{n}\left(\begin{array}{c}
n-1 \\
k-1
\end{array}\right) B_{n-k}^{(n)} \frac{\log \left(1+\lambda\left(e^{t}-1\right)\right)}{\lambda t} x^{k} \\
=\sum_{k=1}^{n}\left(\begin{array}{c}
n-1 \\
k-1
\end{array}\right) B_{n-k}^{(n)} \sum_{l=0}^{k} \sum_{m=0}^{k-l} \sum_{j=0}^{m} \frac{1}{k+1}\left(\begin{array}{c}
k+1 \\
m+1
\end{array}\right)\left(\begin{array}{c}
m+1 \\
j
\end{array}\right) S_{2}(k-m, l) D_{l} \lambda^{l} x^{j} \\
=\sum_{k=0}^{n} \sum_{l=0}^{k} \sum_{m=0}^{k-l} \sum_{j=0}^{m} \frac{1}{k+1}\left(\begin{array}{c}
n-1 \\
k-1
\end{array}\right)\left(\begin{array}{c}
k+1 \\
m+1
\end{array}\right)\left(\begin{array}{c}
m+1 \\
j
\end{array}\right) S_{2}(k-m, l) \lambda^{l} D_{l} B_{n-k}^{(n)} x^{j} \\
=\sum_{j=0}^{n}\left(\sum_{k=j}^{n} \sum_{l=0}^{k-j} \sum_{m=j}^{k-l} \frac{1}{k+1}\left(\begin{array}{c}
n-1 \\
k-1
\end{array}\right)\left(\begin{array}{c}
k+1 \\
m+1
\end{array}\right)\left(\begin{array}{c}
m+1 \\
j
\end{array}\right) S_{2}(k-m, l) \lambda^{l} D_{l} B_{n-k}^{(n)}\right) x^{j} .
\end{aligned}
$$


From (2.3), we note that

$$
x^{n} \sim(1, t), \quad \frac{e^{\lambda t}-1}{\log \left(1+\lambda\left(e^{t}-1\right)\right)} K_{n, 4}(x \mid \lambda) \sim\left(1, e^{t}-1\right) .
$$

For $n \geq 1$, by (2.31), we get

$$
\begin{aligned}
\frac{e^{\lambda t}-1}{\log \left(1+\lambda\left(e^{t}-1\right)\right)} K_{n, 4}(x \mid \lambda) & =x\left(\frac{t}{e^{t}-1}\right)^{n} x^{-1} x^{n}=x B_{n-1}^{(n)}(x) \\
& =\sum_{k=1}^{n}\left(\begin{array}{c}
n-1 \\
k-1
\end{array}\right) B_{n-k}^{(n)} x^{k} .
\end{aligned}
$$

Thus, by (2.32), we have

$$
\begin{aligned}
& K_{n, 4}(x \mid \lambda) \\
& =\sum_{k=1}^{n}\left(\begin{array}{c}
n-1 \\
k-1
\end{array}\right) B_{n-k}^{(n)} \frac{\log \left(1+\lambda\left(e^{t}-1\right)\right)}{e^{\lambda t}-1} x^{k} \\
& =\sum_{k=1}^{n}\left(\begin{array}{c}
n-1 \\
k-1
\end{array}\right) B_{n-k}^{(n)} \sum_{l=0}^{k} \sum_{m=0}^{k-l} \sum_{j=0}^{m} \sum_{i=0}^{j} \frac{1}{j-i+1}\left(\begin{array}{c}
k \\
m
\end{array}\right) \\
& \times\left(\begin{array}{c}
m \\
j
\end{array}\right)\left(\begin{array}{c}
j \\
i
\end{array}\right) \lambda^{l+m-j} S_{2}(k-m, l) D_{l} B_{m-j} x^{i} \\
& =\sum_{i=0}^{n}\left(\sum_{k=i}^{n} \sum_{l=0}^{k-i} \sum_{m=i}^{k-l} \sum_{j=i}^{m} \frac{1}{j-i+1}\left(\begin{array}{c}
n-1 \\
k-1
\end{array}\right)\left(\begin{array}{c}
k \\
m
\end{array}\right)\left(\begin{array}{c}
m \\
j
\end{array}\right)\left(\begin{array}{c}
j \\
i
\end{array}\right)\right. \\
& \left.\times \lambda^{l+m-j} S_{2}(k-m, l) D_{l} B_{m-j} B_{n-k}^{(n)}\right) x^{i} \\
& =\sum_{i=0}^{n}\left(\sum_{k=i}^{n} \sum_{l=0}^{k-i} \sum_{m=i}^{k-l} \sum_{j=i}^{m} \frac{1}{k+1}\left(\begin{array}{c}
n-1 \\
k-1
\end{array}\right)\left(\begin{array}{c}
k+1 \\
m+1
\end{array}\right)\left(\begin{array}{c}
m+1 \\
j+1
\end{array}\right)\right. \\
& \left.\times\left(\begin{array}{c}
j+1 \\
i
\end{array}\right) \lambda^{l+m-j} S_{2}(k-m, l) D_{l} B_{m-j} B_{n-k}^{(n)}\right) x^{i} .
\end{aligned}
$$

Therefore, by (2.30) and (2.33), we obtain the following theorem.

Theorem 6 For $n \geq 0$, we have

$$
\begin{aligned}
& K_{n, 3}(x \mid \lambda) \\
& \quad=\sum_{j=0}^{n}\left(\sum_{k=j}^{n} \sum_{l=0}^{k-j} \sum_{m=j}^{k-l} \frac{1}{k+1}\left(\begin{array}{c}
n-1 \\
k-1
\end{array}\right)\left(\begin{array}{c}
k+1 \\
m+1
\end{array}\right)\left(\begin{array}{c}
m+1 \\
j
\end{array}\right) S_{2}(k-m, l) \lambda^{l} D_{l} B_{n-k}^{(n)}\right) x^{j}
\end{aligned}
$$

and

$$
\begin{aligned}
& K_{n, 4}(x \mid \lambda) \\
& =\sum_{i=0}^{n}\left(\sum_{k=i}^{n} \sum_{l=0}^{k-i} \sum_{m=i}^{k-l} \sum_{j=i}^{m} \frac{1}{k+1}\left(\begin{array}{c}
n-1 \\
k-1
\end{array}\right)\left(\begin{array}{c}
k+1 \\
m+1
\end{array}\right)\left(\begin{array}{c}
m+1 \\
j+1
\end{array}\right)\right. \\
& \left.\times\left(\begin{array}{c}
j+1 \\
i
\end{array}\right) \lambda^{l+m-j} S_{2}(k-m, l) D_{l} B_{m-j} B_{n-k}^{(n)}\right) x^{i}
\end{aligned}
$$


For $s_{n}(x) \sim(g(t), f(t))$, we note that Sheffer identity is given by

$$
s_{n}(x+y)=\sum_{j=0}^{n}\left(\begin{array}{l}
n \\
j
\end{array}\right) s_{j}(x) p_{n-j}(y), \quad \text { where } p_{n}(x)=g(t) s_{n}(x) .
$$

By (2.2) and (2.34), we get

$$
K_{n, 3}(x+y \mid \lambda)=\sum_{j=0}^{n}\left(\begin{array}{l}
n \\
j
\end{array}\right) K_{j, 3}(x \mid \lambda)(y)_{n-j}
$$

where $p_{n}(x)=\frac{\lambda t}{\log \left(1+\lambda\left(e^{t}-1\right)\right)} K_{n, 3}(x \mid \lambda)=(x)_{n}, \quad(n \geq 0)$.

From (2.3) and (2.34), we have

$$
K_{n, 4}(x+y \mid \lambda)=\sum_{j=0}^{n}\left(\begin{array}{l}
n \\
j
\end{array}\right) K_{j, 4}(x \mid \lambda)(y)_{n-j}
$$

where $p_{n}(x)=\frac{e^{\lambda t}-1}{\log \left(1+\lambda\left(e^{t}-1\right)\right)} K_{n, 4}(x \mid \lambda)=(x)_{n}$.

By (1.10), we see that

$$
\left(e^{t}-1\right) K_{n, 3}(x \mid \lambda)=n K_{n-1,3}(x \mid \lambda),
$$

and

$$
\begin{aligned}
\left(e^{t}-1\right) K_{n, 3}(x \mid \lambda) & =e^{t} K_{n, 3}(x \mid \lambda)-K_{n, 3}(x \mid \lambda) \\
& =K_{n, 3}(x+1 \mid \lambda)-K_{n, 3}(x \mid \lambda) .
\end{aligned}
$$

From (2.37) and (2.38), we have

$$
n K_{n-1,3}(x \mid \lambda)=K_{n, 3}(x+1 \mid \lambda)-K_{n, 3}(x \mid \lambda) .
$$

By (1.10) and (2.3), we get

$$
\left(e^{t}-1\right) K_{n, 4}(x \mid \lambda)=n K_{n-1,4}(x \mid \lambda) .
$$

Thus, by (2.40), we have

$$
K_{n, 4}(x+1 \mid \lambda)-K_{n, 4}(x \mid \lambda)=n K_{n-1,4}(x \mid \lambda) .
$$

Therefore, by (2.35), (2.36), (2.39) and (2.41), we obtain the following theorem.

Theorem 7 For $n \geq 0$, we have

$$
\begin{aligned}
& K_{n, 3}(x+y \mid \lambda)=\sum_{j=0}^{n}\left(\begin{array}{c}
n \\
j
\end{array}\right) K_{j, 3}(x \mid \lambda)(y)_{n-j}, \\
& K_{n, 4}(x+y \mid \lambda)=\sum_{j=0}^{n}\left(\begin{array}{c}
n \\
j
\end{array}\right) K_{j, 4}(x \mid \lambda)(y)_{n-j}, \\
& n K_{n-1,3}(x \mid \lambda)=K_{n, 3}(x+1 \mid \lambda)-K_{n, 3}(x \mid \lambda),
\end{aligned}
$$

and 


$$
n K_{n-1,4}(x \mid \lambda)=K_{n, 4}(x+1 \mid \lambda)-K_{n, 4}(x \mid \lambda) .
$$

For $s_{n}(x) \sim(g(t), f(t))$, we note that

$$
\frac{d}{d x} s_{n}(x)=\sum_{l=0}^{n-1}\left(\begin{array}{c}
n \\
l
\end{array}\right)\left\langle\bar{f}(t) \mid x^{n-l}\right\rangle s_{l}(x) .
$$

For $K_{n, 3}(x \mid \lambda) \sim\left(\frac{\lambda t}{\log \left(1+\lambda\left(e^{t}-1\right)\right)}, e^{t}-1\right)$, by (2.42), we get

$$
\begin{aligned}
\left\langle\bar{f}(t) \mid x^{n-l}\right\rangle & =\left\langle\log (1+t) \mid x^{n-l}\right\rangle \\
& =\left\langle\sum_{m=1}^{\infty}(-1)^{m-1}(m-1) ! \frac{t^{m}}{m !} \mid x^{n-l}\right\rangle \\
& =(-1)^{n-l-1}(n-l-1) !
\end{aligned}
$$

Thus, by (2.42) and (2.43), we have

$$
\begin{aligned}
\frac{d}{d x} K_{n, 3}(x \mid \lambda) & =\sum_{l=0}^{n-1}\left(\begin{array}{c}
n \\
l
\end{array}\right)(-1)^{n-l-1}(n-l-1) ! K_{l, 3}(x \mid \lambda) \\
& =n ! \sum_{l=0}^{n-1} \frac{(-1)^{n-l-1}}{l !(n-l)} K_{l, 3}(x \mid \lambda) .
\end{aligned}
$$

By the same method as (2.44), we get

$$
\frac{d}{d x} K_{n, 4}(x \mid \lambda)=n ! \sum_{l=0}^{n-1} \frac{(-1)^{n-l-1}}{l !(n-l)} K_{l, 4}(x \mid \lambda) .
$$

Therefore, by (2.44) and (2.45), we obtain the following theorem.

Theorem 8 For $n \geq 1$, we have

$$
\frac{d}{d x} K_{n, 3}(x \mid \lambda)=n ! \sum_{l=0}^{n-1} \frac{(-1)^{n-l-1}}{l !(n-l)} K_{l, 3}(x \mid \lambda)
$$

and

$$
\frac{d}{d x} K_{n, 4}(x \mid \lambda)=n ! \sum_{l=0}^{n-1} \frac{(-1)^{n-l-1}}{l !(n-l)} K_{l, 4}(x \mid \lambda) .
$$

Let $n \geq 1$. Then, by (1.6), (2.1) and (2.3), we get

$$
\begin{aligned}
K_{n, 3}(y \mid \lambda) \\
=\left\langle\frac{\log (1+\lambda t)}{\lambda \log (1+t)}(1+t)^{y} \mid x^{n}\right\rangle \\
=\left\langle\partial_{t}\left(\frac{\log (1+\lambda t)}{\lambda \log (1+t)}(1+t)^{y}\right) \mid x^{n-1}\right\rangle \\
=\left\langle\frac{\log (1+\lambda t)}{\lambda \log (1+t)} \partial_{t}(1+t)^{y} \mid x^{n-1}\right\rangle+\left\langle\left(\partial_{t} \frac{\log (1+\lambda t)}{\log (1+t)}\right)(1+t)^{y} \mid x^{n-1}\right\rangle .
\end{aligned}
$$


We observe that

$$
\begin{aligned}
\left\langle\frac{\log (1+\lambda t)}{\lambda \log (1+t)} \partial_{t}(1+t)^{y} \mid x^{n-1}\right\rangle & =y\left\langle\frac{\log (1+\lambda t)}{\lambda \log (1+t)}(1+t)^{y-1} \mid x^{n-1}\right\rangle \\
& =y K_{n-1,3}(y-1 \mid \lambda)
\end{aligned}
$$

and

$$
\begin{aligned}
\partial_{t}\left(\frac{\log (1+\lambda t)}{\lambda \log (1+t)}\right) & =\frac{\frac{\lambda}{1+\lambda t} \cdot \lambda \log (1+t)-\log (1+\lambda t) \frac{\lambda}{1+t}}{(\lambda \log (1+t))^{2}} \\
& =\frac{t}{\log (1+t)} \frac{1}{t}\left\{\frac{1}{1+\lambda t}-\frac{\log (1+\lambda t)}{\lambda \log (1+t)}(1+t)^{-1}\right\} .
\end{aligned}
$$

By (2.48), we get

$$
\begin{aligned}
\langle & \left(\partial_{t}\left(\frac{\log (1+\lambda t)}{\log (1+t)}\right)\right)(1+t)^{y}\left|x^{n-1}\right\rangle \\
& =\left\langle\frac{t}{\log (1+t)} \frac{1}{t}\left\{\frac{1}{1+\lambda t}-\frac{\log (1+\lambda t)}{\lambda \log (1+t)}(1+t)^{-1}\right\}(1+t)^{y} \mid x^{n-1}\right\rangle \\
& =\frac{1}{n}\left\langle\left\{\frac{1}{1+\lambda t}-\frac{\log (1+\lambda t)}{\lambda \log (1+t)}(1+t)^{-1}\right\}(1+t)^{y} \mid \frac{t}{\log (1+t)} x^{n}\right\rangle \\
= & \frac{1}{n}\left\langle\left\{\frac{1}{1+\lambda t}-\frac{\log (1+\lambda t)}{\lambda \log (1+t)}(1+t)^{-1}\right\}(1+t)^{y} \mid \sum_{l=0}^{\infty} b_{l} \frac{t^{l}}{l !} x^{n}\right\rangle \\
= & \frac{1}{n} \sum_{l=0}^{n}\left(\begin{array}{c}
n \\
l
\end{array}\right) b_{l}\left\{\left\langle\frac{1}{1+\lambda t}(1+t)^{y} \mid x^{n-l}\right\rangle-\left\langle\frac{\log (1+\lambda t)}{\lambda \log (1+t)}(1+t)^{y-1} \mid x^{n-l}\right\rangle\right\} \\
= & \frac{1}{n} \sum_{l=0}^{n}\left(\begin{array}{c}
n \\
l
\end{array}\right) b_{l}\left\{\left\langle(1+t)^{y} \mid \sum_{m=0}^{\infty}(-\lambda t)^{m} x^{n-l}\right\rangle-K_{n-l, 3}(y-1 \mid \lambda)\right\} \\
= & \frac{1}{n} \sum_{l=0}^{n}\left(\begin{array}{c}
n \\
l
\end{array}\right) b_{l}\left\{\sum_{m=0}^{n-l}(-\lambda)^{m}(n-l)_{m}\left\langle(1+t)^{y} \mid x^{n-l-m}\right\rangle-K_{n-l, 3}(y-1 \mid \lambda)\right\} \\
= & \frac{1}{n} \sum_{l=0}^{n}\left(\begin{array}{c}
n \\
l
\end{array}\right) b_{l}\left\{\sum_{m=0}^{n-l}(-\lambda)^{m}(n-l)_{m}(y)_{n-l-m}-K_{n-l, 3}(y-1 \mid \lambda)\right\} .
\end{aligned}
$$

For $n \geq 1$, from (2.46), (2.47) and (2.49), we have

$$
\begin{aligned}
& K_{n, 3}(x \mid \lambda)-x K_{n-1,3}(x-1 \mid \lambda) \\
& =\frac{1}{n} \sum_{l=0}^{n}\left(\begin{array}{c}
n \\
l
\end{array}\right) b_{l}\left\{\sum_{m=0}^{n-l}(-\lambda)^{m}(n-l)_{m}(x)_{n-l-m}-K_{n-l, 3}(x-1 \mid \lambda)\right\} .
\end{aligned}
$$

Therefore, by (2.50), we obtain the following theorem. 
Theorem 9 For $n \geq 1$, we have

$$
\begin{aligned}
& K_{n, 3}(x \mid \lambda)-x K_{n-1,3}(x-1 \mid \lambda) \\
& \quad=\frac{1}{n} \sum_{l=0}^{n}\left(\begin{array}{c}
n \\
l
\end{array}\right) b_{l}\left\{\sum_{m=0}^{n-l}(-\lambda)^{m}(n-l)_{m}(x)_{n-l-m}-K_{n-l, 3}(x-1 \mid \lambda)\right\} .
\end{aligned}
$$

Remark We note that

$$
\begin{aligned}
b_{n}(x)-x b_{n-1}(x-1) & =\lim _{\lambda \rightarrow 0}\left(K_{n, 3}(x \mid \lambda)-x K_{n-1,3}(x-1 \mid \lambda)\right) \\
& =\frac{1}{n} \sum_{l=0}^{n}\left(\begin{array}{c}
n \\
l
\end{array}\right) b_{l}\left((x)_{n-l}-b_{n-l}(x-1)\right) .
\end{aligned}
$$

From (1.6) and (2.2), we have

$$
\begin{aligned}
K_{n, 4}(y \mid \lambda)= & \left\langle\frac{\log (1+\lambda t)}{(1+t)^{\lambda}-1}(1+t)^{y} \mid x^{n}\right\rangle \\
= & \left\langle\partial_{t}\left(\frac{\log (1+\lambda t)}{(1+t)^{\lambda}-1}(1+t)^{y}\right) \mid x^{n-1}\right\rangle \\
= & \left\langle\frac{\log (1+\lambda t)}{(1+t)^{\lambda}-1} \partial_{t}(1+t)^{y} \mid x^{n-1}\right\rangle \\
& +\left\langle\left(\partial_{t}\left(\frac{\log (1+\lambda t)}{(1+t)^{\lambda}-1}\right)\right)(1+t)^{y} \mid x^{n-1}\right\rangle
\end{aligned}
$$

where $n \geq 1$.

We note that

$$
\begin{aligned}
& \left\langle\frac{\log (1+\lambda t)}{(1+t)^{\lambda}-1}\left(\partial_{t}(1+t)^{y}\right) \mid x^{n-1}\right\rangle \\
& \quad=y\left\langle\frac{\log (1+\lambda t)}{(1+t)^{\lambda}-1}(1+t)^{y-1} \mid x^{n-1}\right\rangle \\
& \quad=y K_{n-1,4}(y-1 \mid \lambda) .
\end{aligned}
$$

Now, we observe that

$$
\begin{aligned}
\partial_{t}( & \left.\frac{\log (1+\lambda t)}{(1+t)^{\lambda}-1}\right) \\
& =\frac{\frac{\lambda}{1+\lambda t}\left((1+t)^{\lambda}-1\right)-\log (1+\lambda t) \lambda(1+t)^{\lambda-1}}{\left((1+t)^{\lambda}-1\right)^{2}} \\
& =\frac{\lambda t}{(1+t)^{\lambda}-1} \frac{1}{t}\left\{\frac{1}{1+\lambda t}-\frac{\log (1+\lambda t)}{(1+t)^{\lambda}-1}(1+t)^{\lambda-1}\right\} .
\end{aligned}
$$

Thus, by (2.53), we get 


$$
\begin{aligned}
\langle & \left(\partial_{t} \frac{\log (1+\lambda t)}{(1+t)^{\lambda}-1}\right)(1+t)^{y}\left|x^{n-1}\right\rangle \\
= & \left\langle\frac{\lambda t}{(1+t)^{\lambda}-1} \frac{1}{t}\left\{\frac{1}{1+\lambda t}-\frac{\log (1+\lambda t)}{(1+t)^{\lambda}-1}(1+t)^{\lambda-1}\right\}(1+t)^{y} \mid x^{n-1}\right\rangle \\
= & \frac{1}{n}\left\langle\frac{\lambda t}{(1+t)^{\lambda}-1}\left\{\frac{1}{1+\lambda t}-\frac{\log (1+\lambda t)}{(1+t)^{\lambda}-1}(1+t)^{\lambda-1}\right\}(1+t)^{y} \mid x^{n}\right\rangle \\
= & \frac{1}{n}\left\langle\left\{\frac{1}{1+\lambda t}-\frac{\log (1+\lambda t)}{(1+t)^{\lambda}-1}(1+t)^{\lambda-1}\right\}(1+t)^{y} \mid \frac{\lambda t}{(1+t)^{\lambda}-1} x^{n}\right\rangle \\
= & \frac{1}{n}\left\langle\left\{\frac{1}{1+\lambda t}-\frac{\log (1+\lambda t)}{(1+t)^{\lambda}-1}(1+t)^{\lambda-1}\right\}(1+t)^{y} \mid \sum_{l=0}^{\infty} K_{l}(\lambda) \frac{t^{l}}{l !} x^{n}\right\rangle \\
= & \frac{1}{n} \sum_{l=0}^{n}\left(\begin{array}{c}
n \\
l
\end{array}\right) K_{l}(\lambda)\left\langle\frac{1}{1+\lambda t}(1+t)^{y} \mid x^{n-l}\right\rangle \\
& -\left\langle\frac{\log (1+\lambda t)}{(1+t)^{\lambda}-1}(1+t)^{y+\lambda-1} \mid x^{n-l}\right\rangle \\
= & \frac{1}{n} \sum_{l=0}^{n}\left(\begin{array}{c}
n \\
l
\end{array}\right) K_{l}(\lambda)\left\{\left\langle(1+t)^{y} \mid \sum_{m=0}^{\infty}(-\lambda t)^{m} x^{n-l}\right\rangle-K_{n-1,4}(y+\lambda-1 \mid \lambda)\right\} \\
= & \frac{1}{n} \sum_{l=0}^{n}\left(\begin{array}{c}
n \\
l
\end{array}\right) K_{l}(\lambda)\left\{\sum_{m=0}^{n-l}(-\lambda)^{m}(n-l)_{m}\left\langle(1+t)^{y} \mid x^{n-l-m}\right\rangle\right. \\
& \left.-K_{n-1,4}(y+\lambda-1 \mid \lambda)\right\} \\
= & \frac{1}{n} \sum_{l=0}^{n}\left(\begin{array}{c}
n \\
l
\end{array}\right) K_{l}(\lambda)\left\{\sum_{m=0}^{n-l}(-\lambda)^{m}(n-l)_{m}(y)_{n-l-m}-K_{n-l, 4}(y+\lambda-1 \mid \lambda)\right\} \\
= & \frac{1}{n} \sum_{l=0}^{n}\left(\begin{array}{c}
n \\
l
\end{array}\right) K_{l}(\lambda)\left\{\sum_{m=0}^{n-l}(-\lambda)^{m}(n-l)_{m}(y)_{n-l-m}-K_{n-l, 4}(y+\lambda-1 \mid \lambda)\right\} .
\end{aligned}
$$

From (2.51), (2.52) and (2.54), we have

$$
\begin{aligned}
& K_{n, 4}(x \mid \lambda)-x K_{n-1,4}(x-1 \mid \lambda) \\
& \quad=\frac{1}{n} \sum_{l=0}^{n}\left(\begin{array}{l}
n \\
l
\end{array}\right) K_{l}(\lambda)\left\{\sum_{m=0}^{n-l}(-\lambda)^{m}(n-l)_{m}(x)_{n-l-m}-K_{n-l, 4}(x+\lambda-1 \mid \lambda)\right\} .
\end{aligned}
$$

Therefore, by (2.55), we obtain the following theorem.

Theorem 10 For $n \geq 1$, we have

$$
\begin{aligned}
& K_{n, 4}(x \mid \lambda)-x K_{n-1,4}(x-1 \mid \lambda) \\
& \quad=\frac{1}{n} \sum_{l=0}^{n}\left(\begin{array}{c}
n \\
l
\end{array}\right) K_{l}(\lambda)\left\{\sum_{m=0}^{n-l}(-\lambda)^{m}(n-l)_{m}(x)_{n-l-m}-K_{n-l, 4}(x+\lambda-1 \mid \lambda)\right\} .
\end{aligned}
$$

Let us consider the following two Sheffer sequences:

$$
\begin{aligned}
K_{n, 3}(x \mid \lambda) & \sim\left(\frac{\lambda t}{\log \left(1+\lambda\left(e^{t}-1\right)\right)}, e^{t}-1\right), \\
(x)^{(n)}=x(x+1) \cdots(x+(n-1)) & \sim\left(1,1-e^{-t}\right) .
\end{aligned}
$$


From (1.14) and (1.15), we have

$$
K_{n, 3}(x \mid \lambda)=\sum_{m=0}^{n} C_{n, m}(x)^{(m)},
$$

where

$$
\begin{aligned}
& C_{n, m} \\
& =\frac{1}{m !}\left\langle\frac{\log (1+\lambda t)}{\lambda \log (1+t)}\left(1-\frac{1}{1+t}\right)^{m} \mid x^{n}\right\rangle \\
& =\frac{1}{m !}\left\langle\frac{\log (1+\lambda t)}{\lambda \log (1+t)} \mid \sum_{l=0}^{\infty}(-1)^{l}(m+l-1)_{l} \frac{t^{m+l}}{l !} x^{n}\right\rangle \\
& =\frac{1}{m !} \sum_{l=0}^{n-m}(-1)^{l}(m+l-1)_{l} \frac{(n)_{m+l}}{l !}\left\langle\frac{\log (1+\lambda t)}{\lambda t} \mid \frac{t}{\log (1+t)} x^{n-m-l}\right\rangle \\
& =\frac{1}{m !} \sum_{l=0}^{n-m}(-1)^{l}(m+l-1)_{l} \frac{(n)_{m+l}}{l !}\left\langle\frac{\log (1+\lambda t)}{\lambda t} \mid \sum_{k=0}^{\infty} b_{k} \frac{t^{k}}{k !} x^{n-m-l}\right\rangle \\
& =\sum_{l=0}^{n-m}(-1)^{l} l !\left(\begin{array}{c}
m+l-1 \\
l
\end{array}\right)\left(\begin{array}{c}
n \\
m+l
\end{array}\right)\left(\begin{array}{c}
m+l \\
m
\end{array}\right) \\
& \times \sum_{k=0}^{n-m-l}\left(\begin{array}{c}
n-m-l \\
k
\end{array}\right) b_{k}\left\langle\frac{\log (1+\lambda t)}{\lambda t} \mid x^{n-m-l-k}\right\rangle \\
& =\sum_{l=0}^{n-m} \sum_{k=0}^{n-m-l}(-1)^{l} l !\left(\begin{array}{c}
m+l-1 \\
l
\end{array}\right)\left(\begin{array}{c}
n \\
m+l
\end{array}\right)\left(\begin{array}{c}
m+l \\
m
\end{array}\right) \\
& \times\left(\begin{array}{c}
n-m-l \\
k
\end{array}\right) b_{k} D_{n-m-l-k} \lambda^{n-m-l-k} \\
& =\sum_{l=0}^{n-m} \sum_{k=0}^{n-m-l}(-1)^{l} l !\left(\begin{array}{c}
m+l-1 \\
l
\end{array}\right)\left(\begin{array}{c}
n \\
m+l
\end{array}\right)\left(\begin{array}{c}
m+l \\
m
\end{array}\right) \\
& \times\left(\begin{array}{c}
n-m-l \\
k
\end{array}\right) b_{n-m-l-k} D_{k} \lambda^{k}
\end{aligned}
$$

Therefore, by (2.57) and (2.58), we obtain the following theorem.

Theorem 11 For $n \geq 0$, we have

$$
\begin{aligned}
& K_{n, 3}(x \mid \lambda) \\
& =\sum_{m=0}^{n}\left\{\sum_{l=0}^{n-m} \sum_{k=0}^{n-m-l}(-1)^{l} l !\left(\begin{array}{c}
m+l-1 \\
l
\end{array}\right)\left(\begin{array}{c}
n \\
m+l
\end{array}\right)\right. \\
& \left.\quad \times\left(\begin{array}{c}
m+l \\
m
\end{array}\right)\left(\begin{array}{c}
n-m-l \\
k
\end{array}\right) b_{n-m-l-k} D_{k} \lambda^{k}\right\}(x)^{(m)} .
\end{aligned}
$$

For $K_{n, 4}(x \mid \lambda) \sim\left(\frac{e^{\lambda t}-1}{\log \left(1+\lambda\left(e^{t}-1\right)\right)}, e^{t}-1\right),(x)^{(n)} \sim\left(1,1-e^{-t}\right)$, we have

$$
K_{n, 4}(x \mid \lambda)=\sum_{m=0}^{n} C_{n, m}(x)^{(m)},
$$


where

$$
\begin{aligned}
C_{n, m} & =\frac{1}{m !}\left\langle\frac{\log (1+\lambda t)}{(1+t)^{\lambda}-1}\left(1-\frac{1}{1+t}\right)^{m} \mid x^{n}\right\rangle \\
= & \frac{1}{m !}\left\langle\frac{\log (1+\lambda t)}{(1+t)^{\lambda}-1} \mid \sum_{l=0}^{\infty}(-1)^{l}(m+l-1)_{l} \frac{t^{m+l}}{l !} x^{n}\right\rangle \\
= & \frac{1}{m !} \sum_{l=0}^{n-m}(-1)^{l}(m+l-1)_{l} \frac{(n)_{m+l}}{l !}\left\langle\frac{\log (1+\lambda t)}{\lambda t} \mid \frac{\lambda t}{(1+t)^{\lambda}-1} x^{n-m-l}\right\rangle \\
= & \frac{1}{m !} \sum_{l=0}^{n-m}(-1)^{l}(m+l-1)_{l} \frac{(n)_{m+l}}{l !}\left\langle\frac{\log (1+\lambda t)}{\lambda t} \mid \sum_{k=0}^{\infty} K_{k}(\lambda) \frac{t^{k}}{k !} x^{n-m-l}\right\rangle \\
= & \frac{1}{m !} \sum_{l=0}^{n-m}(-1)^{l}(m+l-1)_{l} \frac{(n)_{m+l}}{l !} \sum_{k=0}^{n-m-l}\left(\begin{array}{c}
n-m-l \\
k
\end{array}\right) K_{k}(\lambda) \\
& \times\left\langle\frac{\log (1+\lambda t)}{\lambda t} \mid x^{n-m-l-k}\right\rangle \\
= & \frac{1}{m !} \sum_{l=0}^{n-m}(-1)^{l}(m+l-1)_{l} \frac{(n)_{m+l}}{l !} \\
& \times \sum_{k=0}^{n-m-l}\left(\begin{array}{c}
n-m-l \\
k
\end{array}\right) K_{k}(\lambda) D_{n-m-l-k} \lambda^{n-m-l-k} \\
= & \sum_{l=0}^{n-m} \sum_{k=0}^{n-m-l}(-1)^{l} l !\left(\begin{array}{c}
m+l-1 \\
l
\end{array}\right)\left(\begin{array}{c}
n \\
m+l
\end{array}\right)\left(\begin{array}{c}
m+l \\
m
\end{array}\right) \\
& \times\left(\begin{array}{c}
n-m-l \\
k
\end{array}\right) K_{n-m-l-k}(\lambda) D_{k} \lambda^{k} .
\end{aligned}
$$

Therefore, by (2.59) and (2.60), we obtain the following theorem.

Theorem 12 For $n \geq 0$, we have

$$
\begin{aligned}
& K_{n, 4}(x \mid \lambda) \\
& =\sum_{m=0}^{n}\left\{\sum_{l=0}^{n-m} \sum_{k=0}^{n-m-l}(-1)^{l} l !\left(\begin{array}{c}
m+l-1 \\
l
\end{array}\right)\right. \\
& \left.\quad \times\left(\begin{array}{c}
n \\
m+l
\end{array}\right)\left(\begin{array}{c}
m+l \\
m
\end{array}\right)\left(\begin{array}{c}
n-m-l \\
k
\end{array}\right) K_{n-m-l-k}(\lambda) D_{k} \lambda^{k}\right\}(x)^{(m)} .
\end{aligned}
$$

Let us consider the following two Sheffer sequences:

$$
K_{n, 3}(x \mid \lambda) \sim\left(\frac{\lambda t}{\log \left(1+\lambda\left(e^{t}-1\right)\right)}, e^{t}-1\right), \quad B_{n}^{(s)}(x) \sim\left(\left(\frac{e^{t}-1}{t}\right)^{s}, t\right) .
$$

Note that

$\sum_{n=0}^{\infty} B_{n}^{(s)}(x)=\left(\frac{t}{e^{t}-1}\right)^{s} e^{x t}, \quad$ (see Kim et al. 2015; Sen et al. 2013; Ustinov et al. 2002 ), 
where $B_{n}^{(s)}(x)$ are called the higher-order Bernoulli polynomials.

From (1.14) and (1.15), we have

$$
K_{n, 3}(x \mid \lambda)=\sum_{m=0}^{n} C_{n, m} B_{m}^{(s)}(x)
$$

where

$$
\begin{aligned}
C_{n, m} & =\frac{1}{m !}\left\langle\left(\frac{t}{\log (1+t)}\right)^{s} \frac{\log (1+\lambda t)}{\lambda \log (1+t)}(\log (1+t))^{m} \mid x^{n}\right\rangle \\
& =\left\langle\left(\frac{t}{\log (1+t)}\right)^{s} \frac{\log (1+\lambda t)}{\lambda \log (1+t)} \mid \frac{1}{m !}(\log (1+t))^{m} x^{n}\right\rangle \\
& =\left\langle\left(\frac{t}{\log (1+t)}\right)^{s} \frac{\log (1+\lambda t)}{\lambda \log (1+t)} \mid \sum_{l=m}^{\infty} S_{1}(l, m) \frac{t^{l}}{l !} x^{n}\right\rangle \\
& =\sum_{l=m}^{n}\left(\begin{array}{c}
n \\
l
\end{array}\right) S_{1}(l, m)\left\langle\left(\frac{t}{\log (1+t)}\right)^{s} \mid \frac{\log (1+\lambda t)}{\lambda \log (1+t)} x^{n-l}\right\rangle \\
& =\sum_{l=m}^{n}\left(\begin{array}{c}
n \\
l
\end{array}\right) S_{1}(l, m)\left\langle\left(\frac{t}{\log (1+t)}\right)^{s} \mid \sum_{k=0}^{\infty} K_{k, 3}(\lambda) \frac{t^{k}}{k !} x^{n-l}\right\rangle \\
& =\sum_{l=m}^{n}\left(\begin{array}{c}
n \\
l
\end{array}\right) S_{1}(l, m) \sum_{k=0}^{n-l}\left(\begin{array}{c}
n-l \\
k
\end{array}\right) K_{k, 3}(\lambda)\left\langle\left(\frac{t}{\log (1+t)}\right)^{s} \mid x^{n-l-k}\right\rangle \\
& =\sum_{l=m}^{n}\left(\begin{array}{c}
n \\
l
\end{array}\right) S_{1}(l, m) \sum_{k=0}^{n-l}\left(\begin{array}{c}
n-l \\
k
\end{array}\right) K_{k, 3}(\lambda) b_{n-l-k}^{(s)} \\
= & \sum_{l=m}^{n} \sum_{k=0}^{n-l}\left(\begin{array}{c}
n \\
l
\end{array}\right)\left(\begin{array}{c}
n-l \\
k
\end{array}\right) S_{1}(l, m) K_{k, 3}(\lambda) b_{n-l-k}^{(s)} .
\end{aligned}
$$

Here, the Bernoulli numbers of the second kind of order $s$ are defined by the generating function

$$
\left(\frac{t}{\log (1+t)}\right)^{s}=\sum_{j=0}^{\infty} b_{j}^{(s)} \frac{t^{j}}{j !}, \quad \text { (see Kim et al. 2015; Roman 1984). }
$$

Therefore, by (2.61) and (2.62), we obtain the following theorem.

Theorem 13 For $n \geq 0$, we have

$$
K_{n, 3}(x \mid \lambda)=\sum_{m=0}^{n}\left(\sum_{l=m}^{n} \sum_{k=0}^{n-l}\left(\begin{array}{c}
n \\
l
\end{array}\right)\left(\begin{array}{c}
n-l \\
k
\end{array}\right) S_{1}(l, m) K_{k, 3}(\lambda) b_{n-l-k}^{(s)}\right) B_{m}^{(s)}(x) .
$$

Remark In a similar manner, one shows that, for $n \geq 0$,

$$
K_{n, 4}(x \mid \lambda)=\sum_{m=0}^{n}\left(\sum_{l=m}^{n} \sum_{k=0}^{n-l}\left(\begin{array}{c}
n \\
l
\end{array}\right)\left(\begin{array}{c}
n-l \\
k
\end{array}\right) S_{1}(l, m) K_{k, 4}(\lambda) b_{n-l-k}^{(s)}\right) B_{m}^{(s)}(x) .
$$


For $\mu \in \mathbb{C}$ with $\mu \neq 1, s \in \mathbb{N}$, the Frobenius-Euler polynomials of order $s$ are defined by the generating function

$$
\left(\frac{1-\mu}{e^{t}-\mu}\right)^{s} e^{x t}=\sum_{n=0}^{\infty} H_{n}^{(s)}(x \mid \mu) \frac{t^{n}}{n !}, \quad(\text { see }[1,16]) .
$$

For $K_{n, 3}(x \mid \lambda) \sim\left(\frac{\lambda t}{\log \left(1+\lambda\left(e^{t}-1\right)\right)}, e^{t}-1\right), H_{n}^{(s)}(x \mid \mu) \sim\left(\left(\frac{e^{t}-\mu}{1-\mu}\right)^{s}, t\right)$, we have

$$
K_{n, 3}(x \mid \lambda)=\sum_{m=0}^{n} C_{n, m} H_{m}^{(s)}(x \mid \mu),
$$

where

$$
\begin{aligned}
& C_{n, m}= \frac{1}{m !}\left\langle\left(\frac{1-\mu+t}{1-\mu}\right)^{s} \frac{\log (1+\lambda t)}{\lambda \log (1+t)}(\log (1+t))^{m} \mid x^{n}\right\rangle \\
&=\left\langle\left(\frac{1-\mu+t}{1-\mu}\right)^{s} \frac{\log (1+\lambda t)}{\lambda \log (1+t)} \mid \frac{1}{m !}(\log (1+t))^{m} x^{n}\right\rangle \\
&=\left\langle\left(\frac{1-\mu+t}{1-\mu}\right)^{s} \frac{\log (1+\lambda t)}{\lambda \log (1+t)} \mid \sum_{l=m}^{\infty} S_{1}(l, m) \frac{t^{l}}{l !} x^{n}\right\rangle \\
&= \sum_{l=m}^{n}\left(\begin{array}{c}
n \\
l
\end{array}\right) S_{1}(l, m)\left\langle\left(\frac{1-\mu+t}{1-\mu}\right)^{s} \mid \frac{\log (1+\lambda t)}{\lambda \log (1+t)} x^{n-l}\right\rangle \\
&= \sum_{l=m}^{n}\left(\begin{array}{c}
n \\
l
\end{array}\right) S_{1}(l, m)\left\langle\left(\frac{1-\mu+t}{1-\mu}\right)^{s} \mid \sum_{k=0}^{\infty} K_{k, 3}(\lambda) \frac{t^{k}}{k !} x^{n-l}\right\rangle \\
&= \sum_{l=m}^{n}\left(\begin{array}{c}
n \\
l
\end{array}\right) S_{1}(l, m) \sum_{k=0}^{n-l}\left(\begin{array}{c}
n-l \\
k
\end{array}\right) K_{k, 3}(\lambda)\left\langle\left(\frac{1-\mu+t}{1-\mu}\right)^{s} \mid x^{n-l-k}\right\rangle \\
&= \frac{1}{(1-\mu)^{s}} \sum_{l=m}^{n}\left(\begin{array}{c}
n \\
l
\end{array}\right) S_{1}(l, m) \sum_{k=0}^{n-l}\left(\begin{array}{c}
n-l \\
k
\end{array}\right) K_{k, 3}(\lambda) \\
& \times \sum_{j=0}^{s}\left(\begin{array}{c}
s \\
j
\end{array}\right)(1-\mu)^{s-j}\left\langle t^{j} \mid x^{n-l-k}\right\rangle \\
&= \frac{1}{(1-\mu)^{s}} \sum_{l=m}^{n}\left(\begin{array}{c}
n \\
l
\end{array}\right) S_{1}(l, m) \sum_{k=0}^{n-l}\left(\begin{array}{c}
n-l \\
k
\end{array}\right) K_{n-l-k, 3}(\lambda) \\
& \times \sum_{j=0}^{s}\left(\begin{array}{c}
s \\
j
\end{array}\right)(1-\mu)^{s-j}\left\langle t^{j} \mid x^{k}\right\rangle \\
&=\left.\frac{1}{(1-\mu)^{s}} \sum_{l=m}^{n}\left(\begin{array}{c}
n \\
l
\end{array}\right) S_{1}(l, m) \sum_{k=0}^{n-l}\left(\begin{array}{c}
n-l \\
k
\end{array}\right) K_{n-l-k, 3}(\lambda) k !\left(\begin{array}{c}
n \\
l
\end{array}\right)\left(\begin{array}{c}
n-l \\
k
\end{array}\right)\left(\begin{array}{c}
s \\
k
\end{array}\right) \frac{k}{(1-\mu)^{k}} S_{1}(l, m) K_{n-l-k, 3}(\lambda) . \mu\right)^{s-k} \\
& k=0
\end{aligned}
$$

Therefore, by (2.63) and (2.64), we obtain the following theorem. 
Theorem 14 For $n \geq 0$, we have

$$
\begin{aligned}
& K_{n, 3}(x \mid \lambda) \\
& \quad=\sum_{m=0}^{n}\left(\sum_{l=m}^{n} \sum_{k=0}^{n-l}\left(\begin{array}{c}
n \\
l
\end{array}\right)\left(\begin{array}{c}
n-l \\
k
\end{array}\right)\left(\begin{array}{c}
s \\
k
\end{array}\right) \frac{k !}{(1-\mu)^{k}} S_{1}(l, m) K_{n-l-k, 3}(\lambda)\right) H_{m}^{(s)}(x \mid \mu) .
\end{aligned}
$$

Remark Proceeding similarly to the above, one can show that, for $n \geq 0$,

$$
\begin{aligned}
& K_{n, 4}(x \mid \lambda) \\
& \quad=\sum_{m=0}^{n}\left(\sum_{l=m}^{n} \sum_{k=0}^{n-l}\left(\begin{array}{c}
n \\
l
\end{array}\right)\left(\begin{array}{c}
n-l \\
k
\end{array}\right)\left(\begin{array}{c}
s \\
k
\end{array}\right) \frac{k !}{(1-\mu)^{k}} S_{1}(l, m) K_{n-l-k, 4}(\lambda)\right) H_{m}^{(s)}(x \mid \mu) .
\end{aligned}
$$

\section{Conclusion}

The first degenerate version of the Bernoulli polynomials of the second kind appeared in the paper by Korobov $(1996,2001)$. Here, we study two degenerate versions of the Bernoulli polynomials of the second kind which will be called Korobov polynomials of third kind and of the fourth kind. Some properties, identities, recurrence relations and connections with other polynomials are investigated by using umbral calculus.

\section{Authors' contributions}

All authors contributed equally to this work. All authors read and approved the final manuscript.

\section{Author details}

${ }^{1}$ Department of Mathematics, College of Science, Tianjin Polytechnic University, Tianjin 300387, China. ${ }^{2}$ Department of Mathematics, Kwangwoon University, Seoul 139-701, Republic of Korea. ${ }^{3}$ Department of Mathematics, Sogang University, Seoul 121-742, Republic of Korea.

\section{Acknowledgements}

The work reported in this paper was conducted during the sabbatical year of Kwangwoon University in 2014.

\section{Competing interests}

The authors declare that they have no competing interests.

Received: 11 August 2015 Accepted: 6 October 2015

Published online: 14 October 2015

\section{References}

Araci S, Acikogz M (2012) A note on the Frobenius-Euler numbers and polynomials associated with Bernstein polynomials. Adv Stud Contemp Math 22(3):399-406

Bayad A, Kim T (2010) Identities for the Bernoulli, the Euler and the Genocchi numbers and polynomials. Adv Stud Contemp Math 20(2):247-253

Carlitz L (1979) Degenerate Stirling, Bernoulli and EEuler numbers. Utilitas Math 15:51-88

Dattoli G, Lorenzutta S, Cesarano C (2006) Bernstein polynomials and operational methods. J Comput Anal Appl 8(4):369-377

Dattoli G, Lorenzutta S, Ricci PE, Cesarano C (2004) On a family of hybrid polynomials. Integral Transforms Spec Funct 15(6):485-490

Kim DS, Kim T, Lee S-H (2015) Higher-order Daehee of the first kind and poly-Cauchy of the first kind mixed type polynomials. J Comput Anal Appl 18(4):699-714

Kim DS, Kim T, Rim S-H, Dolgy DV (2015) Barnes' multiple Bernoulli and Hermite mixed-type polynomials. Proc Jangjeon Math Soc 18(1):7-19

Kim T (2014) Identities involving laguerre polynomials derived from umbral calculus. Russ J Math Phys 21(1):36-45

Kim T, Kim DS, Bayad A, Rim S-H (2012) Identities on the Bernoulli and the Euler numbers and polynomials. Ars Combin 107:455-463

Kim T, Mansour T (2014) Umbral calculus associated with Frobenius-type Euler polynomials. Russ I math Phys 21(4):484-493

Korobov NM (1996) Special polynomials and their applications, diophantine approximations. Math Notes 2:77-89

Korobov NM (2001) On some properties of special polynomials. In: Proceedings of the IV International Conference Modern Problems of Number Theory and its Applications (Russian) (Tula, 2001), vol. 1, pp. 40-49 
Nisar KS, Mondal SR, Agarwal P, Al-Dhaifallah M (2015) The umbral operator and the integration involving generalized Bessel-type functions. Open Math 13(1):426-435

Pylypiv VM, Maliarchuk AR (2014) On some properties of Korobov polynomials. Carpathian Math Publ 6(1):130-133 Roman S (1984) The umbral calculus, Pure and Applied Mathematics, vol 111. Academic Press Inc [Harcourt Brace Jovanovich, Publishers], New York

Sen E (2013) Theorems on Apostol-Euler polynomials of higher order arising from Euler basis. Adv Stud Contemp Math (Kyungshang) 23(2):337-345

Srivastava HM, Nisar KS, Khan MA (2014) Some umbral calculus presentations of the Chan-Chyan-Srivastava polynomials and the Erku-Srivastava polynomials. Proyecciones 33(1):77-90

Ustinov AV (2002) A discrete analogue of Euler's summation formula. Mat Zametki 71(6):931-936

Ustinov AV (2003) Korobov polynomials and umbral analysis. Chebyshevskĭ Sb 4(8):137-152

\section{Submit your manuscript to a SpringerOpen ${ }^{\circ}$} journal and benefit from:

- Convenient online submission

- Rigorous peer review

- Immediate publication on acceptance

- Open access: articles freely available online

- High visibility within the field

- Retaining the copyright to your article

Submit your next manuscript at $\mathbf{s p r i n g e r o p e n . c o m ~}$ 